\title{
One-pot synthesis of novel thioxanthone crown ethers
}

\author{
Hashem Sharghi* and Ali Reza Salimi Beni \\ Department of Chemistry, Shiraz University, Shiraz 71454, I.R. Iran \\ E-mail: shashem@chem.susc.ac.ir
}

\begin{abstract}
The reaction of thiosalicylic acid (TSA) and benzocrown ethers in concentrated sulfuric acid affords a general and efficient way to prepare thioxanthone crown ethers. This chemistry presumably proceeds by intermolecular sulfur electrophilic reaction and subsequently intramolecular electrophilic cyclization. Most of the reactions show high regioselectivity in good yields.
\end{abstract}

Keywords: Thioxanthone, thiosalicylic acid, crown ether

\section{Introduction}

Thioxanthones are an important class of molecules and are a common heterocyclic scaffold in biologically active and medicinally significant compounds. The thioxanthone ring is the core structure of a wide variety of naturally occurring and synthetic compounds that exhibit extraordinary anti-tumor ${ }^{1-3,6,9}$ anti-parasitic ${ }^{4,5}$ and anti-cancer activity ${ }^{6,7,10}$. Thioxanthone derivatives are potential anti-cancer drugs and some thioxanthones containing plant extract are directly used in traditional medicines. ${ }^{5-7}$ Crown ethers have enjoyed widespread use in various areas of science and technology ${ }^{11}$ ever since the first preparation of the ligands by Pederson. ${ }^{12}$

\section{Results and Discussion}

Standard syntheses of the thioxanthones skeleton typically involve multi-step procedures which generally involve the intermediacy of a benzophenone or a diarylthioether, plus harsh reaction conditions, and/or strong acids are often employed. ${ }^{6-8}$ Other methods for the synthesis of thioxanthones are used of concentrated sulfuric acid at room or high temperature. ${ }^{13,14}$ Also we have recently reported preparation of hydroxythioxanthone derivatives and some of their applications. ${ }^{15,16,17}$ 
We now wish to report a novel and efficient one-pot method for synthesis of thioxanthone crown ether under concentrated sulfuric acid at room temperature (Scheme 1).

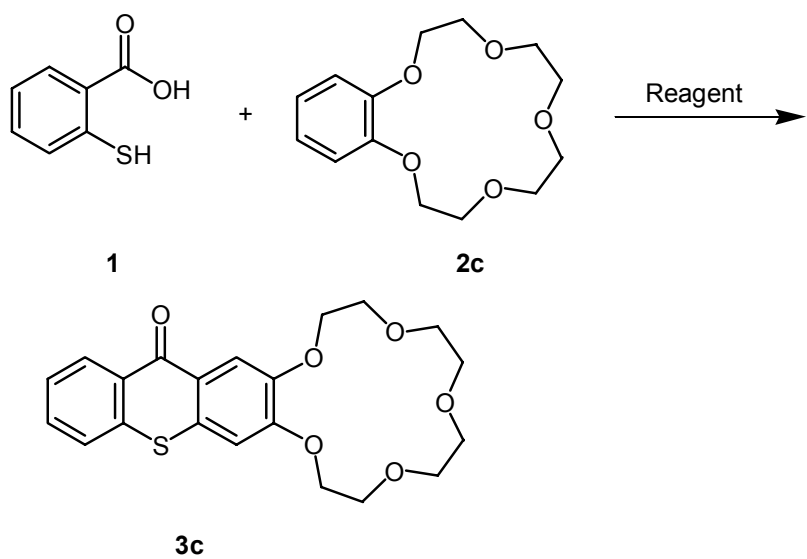

\section{Scheme 1}

To develop an efficient reagent for the synthesis of thioxanthone crown ethers, we initially examined the reaction of thiosalicylic acid (TSA) with benzo-15-crown-5 (2c) in the presence of various reagents. The reaction monitored via TLC (n-hexane $/ \mathrm{CH}_{2} \mathrm{Cl}_{2}$ ) and ${ }^{1} \mathrm{H} \mathrm{NMR}$ spectroscopy (Table 1).

Table 1. The results of the reaction of $1(1 \mathrm{mmol})$ and $2 \mathbf{c}(1 \mathrm{mmol})$ in the presence of various reagents

\begin{tabular}{ccccc}
\hline Entry & Conditions & Time (h) & Temp. ${ }^{\circ} \mathrm{C}$ & Yield (\%) ${ }^{\mathrm{a}}$ \\
\hline 1 & $\mathrm{CH}_{3} \mathrm{SO}_{3} \mathrm{H}$ & 24 & r. t. & - \\
2 & $\mathrm{CH}_{3} \mathrm{SO}_{3} \mathrm{H}$ & 1.5 & 100 & - \\
3 & $\mathrm{CH}_{3} \mathrm{SO}_{3} \mathrm{H}+\mathrm{Al}_{2} \mathrm{O}_{3}{ }^{14}$ & 10 & 110 & - \\
4 & $\mathrm{CH}_{3} \mathrm{SO}_{3} \mathrm{H}+\mathrm{Al}_{2} \mathrm{O}_{3}{ }^{14}$ & 1.5 & 140 & - \\
5 & $\mathrm{H}_{3} \mathrm{PO}_{4}$ & 24 & r. t. & - \\
6 & $\mathrm{PPA}^{6}$ & 24 & 100 & - \\
7 & $\mathrm{PPA}^{2} \mathrm{Al}_{2} \mathrm{O}_{3}$ & 24 & 100 & - \\
8 & $\mathrm{P}_{4} \mathrm{O}_{10}+\mathrm{CH}_{3} \mathrm{SO}_{3} \mathrm{H}$ & 16 & 100 & - \\
9 & $\mathrm{H}_{2} \mathrm{SO}_{4} 70 \%$ & 80 & - \\
10 & $\mathrm{Conc.}_{2} \mathrm{SO}_{4} 98 \%$ & 2 & 0 & 25 \\
11 & Conc. $\mathrm{H}_{2} \mathrm{SO}_{4} 98 \%$ & 2 & r. t. & 40 \\
12 & Conc. $\mathrm{H}_{2} \mathrm{SO}_{4} 100 \%$ & 2 & r. t. & 40 \\
13 & Conc. $\mathrm{H}_{2} \mathrm{SO}_{4} 98 \%{ }^{b}$ & 2 & r. t. & 70 \\
14 & Conc. $\mathrm{H}_{2} \mathrm{SO}_{4} 98 \%$ & 2 & 90 & 30 \\
\hline
\end{tabular}

${ }^{\mathrm{a}}$ Isolated yield, ${ }^{\mathrm{b}}$ ratio 1:7 of TSA/Crown ether were used 
As is shown in Table 1 the best results were obtained using concentrated sulfuric acid at room temperature for 2 hours. The ratio TSA/crown ether is 1:7 (Table 1: Entry 13). No increase in the yields was observed with improving the temperature. To establish the generality and applicability of this method, various crown ethers were subjected to the same reaction conditions to furnish the corresponding thioxanthone crown ethers in good yields (Scheme 2) (Table 2).

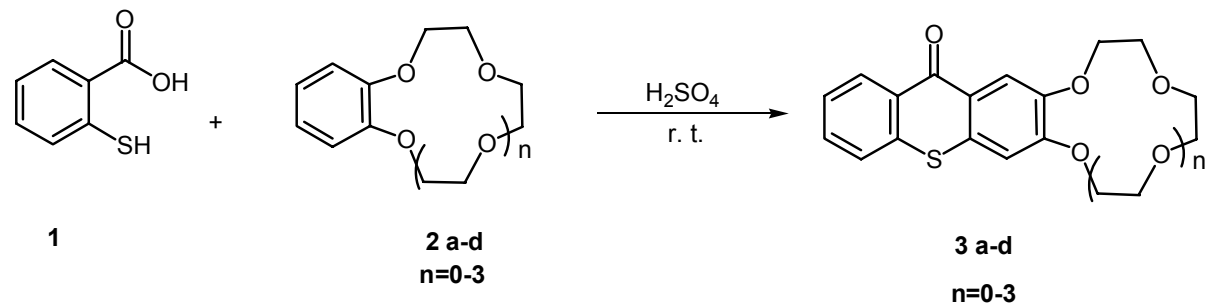

\section{Scheme 2}

Table 2. The results of reaction of $1(1 \mathrm{mmol})$ and $\mathbf{2 a - d}(7 \mathrm{mmol})$ in the presence of concentrated sulfuric acid

\begin{tabular}{ccccc}
\hline Entry & $\mathrm{n}$ & Time/h & Product & Yield $^{\mathrm{a}}(\%)$ \\
\hline 1 & 0 & 2 & $\mathbf{3 a}$ & 70 \\
2 & 1 & 2 & $\mathbf{3 b}$ & 70 \\
3 & 2 & 2 & $\mathbf{3 c}$ & 68 \\
4 & 3 & 2 & $\mathbf{3 d}$ & 63 \\
\hline
\end{tabular}

${ }^{\mathrm{a}}$ Isolated yield

No attempt has been made to probe the mechanism of the reaction. We assume that the mercapto group 1 (TSA) is oxidized to sulfenic acid which immediately decomposes to a sulfenium ion $4^{14 a}$ (Scheme 3). Electrophilic substitution of sulfenium ion with the benzo-15crown-5 (2c) would then give intermediate thioether and the cyclization of thioether gives the thioxanthone-15-crown-5 ether (3c) (Scheme 4). Thioxanthone crown ether yields depended directly upon the sulfuric acid concentration because sulfonation of $\mathbf{2 c}$ competes with the generation of sulfenium ion (4) and subsequent electrophilic substitution ${ }^{14}$.

The experimental procedure for the preparation of thioxanthone crown ethers are remarkably simple and does not require the use of any solvent or inert atmosphere and does not require the purification of products by column chromatography. 


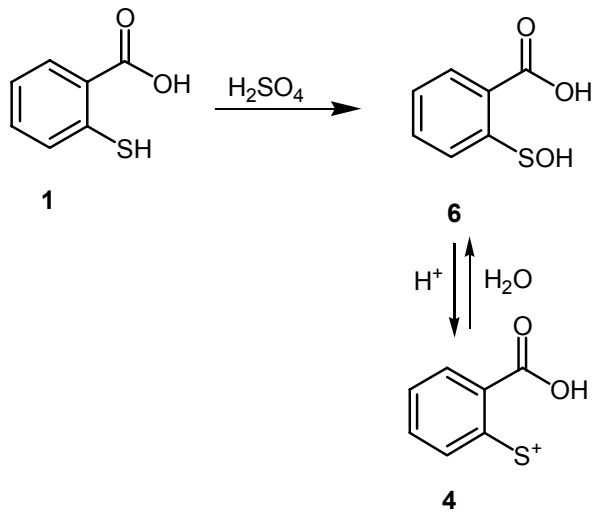

Scheme 3

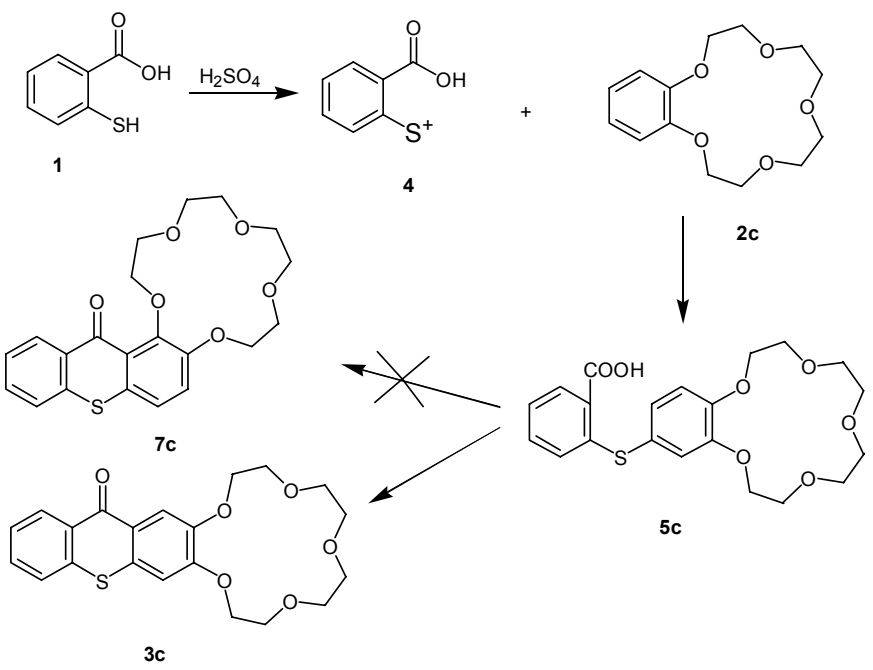

Scheme 4

\section{Experimental Section}

General Procedure. Thiosalicylic acids, benzocrown ethers and sulfuric acid (98\%), were purchased from Fluka and Merck in high purity. The preparation of the benzo-9-crown-3 used as precursors has been described and it was purified by chromatography on silica-gel, eluted with 1:1 $\mathrm{CH}_{2} \mathrm{Cl}_{2}$ : diethyl ether (See reference 18). NMR spectra were recorded in $\mathrm{CDCl}_{3}$ or DMSO$\mathrm{d}_{6}$ on a Bruker Advanced Dpx-250 ( ${ }^{1} \mathrm{H}$ NMR $250 \mathrm{MHz}$ and ${ }^{13} \mathrm{C}$ NMR $\left.62.9 \mathrm{MHz}\right)$ spectrophotometer using TMS as internal standard. Infrared spectra were recorded on a Perkin Elmer IR-157G spectrometer, UV spectra were recorded on a Pharmacia Biotech (Ultrospec 3000) UV/Visible spectrometer and melting points were taken on a Büchi melting apparatus. The TLC was performed on plates coated with silica gel (silica gel $60 \mathrm{GF}_{254}$, Merck). 
Preparation of 2,3,5,6-tetrahydro-1,4,7-benzotrioxonine ${ }^{18}$ (2a). A 2-L round-bottom flask was charged with catechol $(16.3 \mathrm{~g}, 0.148 \mathrm{~mol})$, water $(1700 \mathrm{~mL})$, and LiOH $(12.4 \mathrm{~g}, 0.296 \mathrm{~mol})$, and the mixture was stirred for $5 \mathrm{~min}$. To this was added dropwise 1,5-dichloro-3-oxapentane $(21.7 \mathrm{~g}, 0.148 \mathrm{~mol})$ and the reaction mixture was refluxed for $86 \mathrm{~h}$. Upon cooling, the mixture was acidified to $\mathrm{pH}=2$ by an addition dilute $\mathrm{H}_{2} \mathrm{SO}_{4}$ and were extracted with $\mathrm{CH}_{2} \mathrm{Cl}_{2}(3 \times 100 \mathrm{~mL})$. After washing with $5 \% \mathrm{NaOH}(3 \times 100 \mathrm{~mL})$, saturated $\mathrm{NaCl}(100 \mathrm{~mL})$, and water, the extracts were dried over anhydrous $\mathrm{Na}_{2} \mathrm{~S}_{4}$. After solvent removal, the resulting reddish slurry was purified by column chromatography on silica gel (grade 60, 230-400 mesh). Elution with 1:1 $\mathrm{CH}_{2} \mathrm{Cl}_{2}$ : diethyl ether yielded, in the initial fractions, $8.0 \mathrm{~g}(30 \%)$ of 2,3,5,6-Tetrahydro-1,4,7benzotrioxonine (2a), mp 70-72 ${ }^{\circ} \mathrm{C}$ (lit. ${ }^{18} \mathrm{mp}$ (for protio analog) 69-71 ${ }^{\circ} \mathrm{C}$ ); ${ }^{1} \mathrm{H} \mathrm{NMR}\left(\mathrm{CDCl}_{3} 250\right.$ $\mathrm{MHz}): \delta 3.91(\mathrm{~m}, 4 \mathrm{H}) ; 4.34(\mathrm{~m}, 4 \mathrm{H}) ; 6.98(\mathrm{~m}, 4 \mathrm{H}) ;{ }^{13} \mathrm{C} \mathrm{NMR}\left(\mathrm{CDCl}_{3} 62.9 \mathrm{MHz}\right): \delta$ 74.1, 76.6, 120.7, 124.0, 151.4; IR (KBr, cm $\left.{ }^{-1}\right): 3315$ (s), 1579 (s), 1494 (s), 1446 (s), 1365 (s), 1024 (s), 999 (s), 761 (s), 490 (s); Mass m/z (\%): $180\left(\mathrm{M}^{+}, 40.9\right)$; 136 (100), 80 (38.8), UV $\left(\mathrm{CHCl}_{3}\right) \lambda_{\max } / \mathrm{nm}$ (ع): 241.5 (610), 274.6 (1782); Anal. Calcd for $\mathrm{C}_{10} \mathrm{H}_{12} \mathrm{O}_{3}$ (180.1): Calc. C 66.65\%, H 6.71\%; found C $66.55 \%, \mathrm{H} 6.67 \%$.

General procedure for the synthesis of thioxanthone crown ethers. To a stirring mixture of concentrated sulfuric acid 98\% (2 mL) and thiosalicylic acid (TSA) $(0.154 \mathrm{~g}, 1 \mathrm{mmol})$ in a round-bottomed flask was gradually added $(60 \mathrm{~min})$ the appropriate crown ether $(7 \mathrm{mmol})$ at room temperature and then the mixture was stirred for one hour. The reaction progress was monitored by TLC. After completion of the reaction, the mixture was poured onto ice and the product was filtered, washed with water and then washed with a saturated aqueous solution of sodium hydrogen carbonate or cesium carbonate until alkali-free and dried under vacuum over night at room temperature to yield product as shown in Table 2.

2,3,5,6-Tetrahydro-14H-thioxantheno[2,3-b][1,4,7]trioxonin-14-one (3a). Compound 3a was obtained as yellow crystal in $70 \%$ yield. $\mathrm{Mp}=178-180^{\circ} \mathrm{C},{ }^{1} \mathrm{H}$ NMR $\left(\mathrm{CDCl}_{3}, 250 \mathrm{MHz}\right): \delta 3.91$ $(\mathrm{m}, 4 \mathrm{H}), 4.30(\mathrm{~m}, 2 \mathrm{H}), 4.70(\mathrm{~m}, 2 \mathrm{H}), 7.08(\mathrm{~s}, 1 \mathrm{H}), 7.51(\mathrm{~m}, 3 \mathrm{H}), 8.25(\mathrm{~s}, 1 \mathrm{H}), 8.56(\mathrm{~d}, 1 \mathrm{H}, \mathrm{J}=7.4$ $\mathrm{Hz}) ;{ }^{13} \mathrm{C} \mathrm{NMR}\left(\mathrm{CDCl}_{3}, 62.9 \mathrm{MHz}\right): \delta 71.6,71.8,72.8,76.5$, , 117.2, 124.7, 124.9, 125.8, 126.0, 127.1, 129.7, 131.9, 133.4, 137.1, 150.6, 155.8, and 178.7; IR (KBr, cm $\left.{ }^{-1}\right): 2900$ (s), 1640 (s), 1580 (s), 1490 (s), 1300 (s), 1125 (s), 1030 (s), 880 (s), 740 (s); Mass m/z (\%): 314 (M , 26.5); 270 (17.6), 214 (29.4), 186 (34.8), 158 (25.5) and 57 (100\%); UV $\left(\mathrm{CHCl}_{3}\right) \lambda_{\max } / \mathrm{nm}(\varepsilon): 253.6$ (2029), 318.8 (2537), 363.4 (2446), 395.3 (2420); Anal. Calcd for $\mathrm{C}_{17} \mathrm{H}_{14} \mathrm{O}_{4} \mathrm{~S}$ (314.36): Calc. C $64.95 \%, \mathrm{H} 4.49 \%$; found C $64.72 \%, \mathrm{H} 4.43 \%$

$\mathbf{2 , 3 , 5 , 6 , 8 , 9 - H e x a h y d r o - 1 7 H}$-thioxantheno[2,3-b][1,4,7,10]tetraoxacyclododecin-17-one (3b). Compound $\mathbf{3 b}$ was obtained as yellow crystal in $70 \%$ yield. $\mathrm{Mp}=286-288{ }^{\circ} \mathrm{C}$ (decomposed); ${ }^{1} \mathrm{H}$ NMR (CDCl $3,250 \mathrm{MHz}): \delta 3.79(\mathrm{~s}, 4 \mathrm{H}), 3.86(\mathrm{~m}, 4 \mathrm{H}), 3.93(\mathrm{~m}, 2 \mathrm{H}), 4.30(\mathrm{~s}, 2 \mathrm{H}), 7.05(\mathrm{~s}, 3 \mathrm{H})$, $7.47(\mathrm{t}, 1 \mathrm{H}, \mathrm{J}=7.2 \mathrm{~Hz}), 7.57(\mathrm{~m}, 2 \mathrm{H}), 8.22(\mathrm{~s}, 1 \mathrm{H}), 8.58(\mathrm{~d}, 1 \mathrm{H} \mathrm{J}=7.4 \mathrm{~Hz}) ;{ }^{13} \mathrm{C} \mathrm{NMR}\left(\mathrm{CDCl}_{3}, 62.9\right.$ $\mathrm{MHz}): \delta 69.5,71.0,71.4,72.9,111.9,118.6,124.1,125.8,126.2,128.6,129.7,131.8,132.7$, 137.0, 149.7, 155.4, 178.8; IR (KBr, cm ${ }^{-1}$ ): 2873 (s), 1635 (s), 1593 (s), 1504 (s), 1440 (s), 1292 (s), 1269 (s), 1253 (s), 1147 (s), 1114 (s) 742 (s); Mass m/z (\%): 358 (M+, 4.1); 270 (9.4), 214 (8.2), 186 (7.1), 149 (21.3) and 57 (100); UV ( $\left.\mathrm{CHCl}_{3}\right) \lambda_{\max } / \mathrm{nm}(\varepsilon): 253.5$ (2123), 314.6 (2501), 
368.2 (2565), 389.1 (2373); Anal. Calcd for $\mathrm{C}_{19} \mathrm{H}_{18} \mathrm{O}_{5} \mathrm{~S}$ (358.41): Calc. C 63.67\%, H 5.06\%; found C $63.51 \%$, H $5.13 \%$.

\section{2,3,5,6,8,9,11,12-Octahydro-20H-thioxantheno $[2,3-b][1,4,7,10,13]$ pentaoxacyc-}

lopentadecin-20-one (3c). Compound 3c was obtained as yellow crystal in 68\%.yield. Mp=157$158{ }^{\circ} \mathrm{C} ;{ }^{1} \mathrm{H}$ NMR $\left(\mathrm{CDCl}_{3}\right): \delta 3.34(\mathrm{~s}, 8 \mathrm{H}), 3.64(\mathrm{~s}, 4 \mathrm{H}), 3.82(\mathrm{~s}, 2 \mathrm{H}), 4.20(\mathrm{~s}, 2 \mathrm{H}), 7.34(\mathrm{~s}, 3 \mathrm{H})$, 7.57 (t, 1H, J=7.5 Hz), 7.73 (t, 1H, J=7.5. Hz), 7.81 (d, 1H, J=7.9. Hz), 7.87 (s, 1H), $8.54(\mathrm{~d}, 1 \mathrm{H}$, $\mathrm{J}=7.9 . \mathrm{Hz}) ;{ }^{13} \mathrm{C} \mathrm{NMR}\left(\mathrm{CDCl}_{3}\right): \delta 68.7,68.9,69.0,69.8,69.9,70.9,108.7,111.0,122.4,126.7$, 126.8, 128.2, 129.1, 131.0, 132.5, 136.8, 148.3, 153.5, 177.7; IR (KBr, cm $\left.{ }^{-1}\right): 2873$ (s), 1591 (s), 1506 (s), 1446 (s), 1407 (s), 1261 (s), 1215 (s), 1137 (s), 1083 (s), 935 (s) 740 (s), Mass m/z (\%): $402\left(\mathrm{M}^{+}, 28.3\right) ; 270$ (50.5), 214 (18.7), 97 (10.8) and 43 (100); UV $\left(\mathrm{CHCl}_{3}\right) \lambda_{\max } / \mathrm{nm}(\varepsilon): 253.6$ (2022), 319.3 (2566), 364.3 (2483), 395.0 (2368); Anal. Calcd for $\mathrm{C}_{21} \mathrm{H}_{22} \mathrm{O}_{6} \mathrm{~S}$ (402.46): Calc. C $62.67 \%$, H 5.51\%; found C $63.49 \%$, H 5.46\%.

\section{2,3,5,6,8,9,11,12,14,15-Decahydro-23H-thioxantheno[2,3-b][1,4,7,10,13,16]hexa-}

oxacyclooctadecin-23-one (3d). Compound 3d was obtained as yellow crystal in 63\% yield. $\mathrm{Mp}=289-291{ }^{\circ} \mathrm{C}$ (decomposed); ${ }^{1} \mathrm{H}$ NMR $\left(\mathrm{CDCl}_{3}, 250 \mathrm{MHz}\right): \delta 3.76(\mathrm{~s}, 8 \mathrm{H}), 3.98(\mathrm{~s}, 2 \mathrm{H}), 4.28$ $(\mathrm{s}, 2 \mathrm{H}), 6.91(\mathrm{~s}, 1 \mathrm{H}), 7.50(\mathrm{~m}, 2 \mathrm{H}), 7.57(\mathrm{~s}, 1 \mathrm{H}), 8.03(\mathrm{~s}, 1 \mathrm{H}), 8.61(\mathrm{~d}, 1 \mathrm{H}, \mathrm{J}=7.5 . \mathrm{Hz}) ;{ }^{13} \mathrm{C} \mathrm{NMR}$ $\left(\mathrm{CDCl}_{3}, 62.9 \mathrm{MHz}\right): \delta 67.0,68.4,68.8,68.9,69.0,70.3,70.4,70.5,70.6,70.7$ 107.5, 109.7, 111.2, 115.8, 123.1, 125.8, 126.1, 129.3, 129.7, 131.5, 137.0, 153.0, 178.5; IR (KBr, cm $\left.{ }^{-1}\right): 2923$ (s), 2860 (s), 1681 (s), 1589 (s), 1504 (s), 1411 (s), 1259 (s), 1126 (s), 950 (s), 742 (s); Mass m/z (\%): $446\left(\mathrm{M}^{+}, 12.0\right) ; 270$ (61.7) 214 (26.8), 84 (80.4) and 57 (100); UV $\left(\mathrm{CHCl}_{3}\right) \lambda_{\max } / \mathrm{nm}(\varepsilon)$ : 253.7 (2072), 315.6 (2195), 374.2 (2942), 385.6 (2000); Anal. Calcd for $\mathrm{C}_{23} \mathrm{H}_{26} \mathrm{O}_{7} \mathrm{~S}$ (446.51): Calc. C $61.87 \%$, H 5.87\%; found C $61.98 \%$, H 5.64\%.

\section{Acknowledgements}

We gratefully acknowledge the support of this work by the Shiraz University. We are also grateful to Mr. H. Sajedian Fard and Mr. M. S. Darvish Tafvizi for helpful cooperation.

\section{References}

1. Foster, B. J.; Wiegand, R. A.; Partricia, S. P.; Lorusso, M.; Rake, J.; Corbett, T. H. Clin. Cancer Res. 1997, 3, 2047.

2. Wentland, M. P.; Perni, R. B.; Hlavac, A. G.; Corbett, T. H.; Coughlin, S. A.; Rake, J. B. Bioorg. Med. Chem. Lett. 1994, 4, 609.

3. Lubomir, V.; Foster, H. M. Chem. Abstr. 1979, 90, 72060a.

4. Watanabe, M.; Pate, M.; Tsukazaki, M.; Furukawa, S. Chem. Pharm. Bull. 1989, 37, 36.

5. Archer, S.; Mattoccia, L. P.; Cioli, D.; Seyed-Mozaffari, A.; Zayed, A. H. J. Med. Chem. 1988, 31, 254. 
6. a) Showalter, H. D. H.; Angelo, M. M.; Berman, E. M. J. Med. Chem. 1988, 31, 1527. b) Archer, S.; Abdel-Hadi, Zayed; Rabindra Rej; Thomas A. Rugino. J. Med. Chem. 1983, 26, 1240 .

7. Laidlaw, G. M.; Collins, J. C.; Archer, S.; Rosi, D.; Schulenberg J. W. J. Org. Chem. 1973, $38,1743$.

8. Moon, J-K.; Park, J-W.; Lee, W. S.; Kang, Y-J.; Chung, H-A.; Shin, M-S.; Yoon, Y-J. J. Heterocyclic Chem. 1999, 36, 793.

9. Krapcho, A. P.; Haydar, S. N. J. Heterocyclic Chem. 1997, 34, 1637.

10. Okabayashi, I.; Fujiwara, H. J. Heterocyclic Chem. 1994, 31, 733.

11. (a) Patai, S. In The Chemistry of Ethers, Crown Ethers, Hydroxyl Groups and Their Sulphur Analogues; John Wiley \& Sons: New York, 1981. (b) Javanbakht M.; Ganjali M.; Eshghi H.; Sharghi H.; Shamsipur M. Electroanalysis 1999, 11, 81. (c) Shamsipur M.; Rouhani S.; Sharghi; H.; Ganjali M.; Eshghi H. Anal. Chem. 1999, 71, 4938. (d) Shamsipur M.; Rouhani S.; Ganjali M.; Eshghi H.; Sharghi H. Microchemical Journal 1999 63, 202. (e) Shamsipur M.; Soleymanpour A.; Akhond M.; Sharghi H.; Massah A. Talanta 2002, 58, 237 (f) Marchand A. P.; Lai, H.; Deng B-L.; Calderón J. Arkivoc 2005, 5, 7. (g) Gerencsér J.; Huszthy P.; Nógrádi M. Arkivoc 2004, 7, 7 (h) Xu X-H.; Chen B.; Wu L-Z. ; Zhang L-P.; Tung C-H. Arkivoc 2003 2, 182 (i) Maitra U.; Nath S. Arkivoc 2005, 3,133.

12. (a) Pedersen, C. J. J. Am. Chem. Soc. 1967; 89, 2495.(b) Gokel, G. W.; Leevy, M. W.; Weber, M. E. Chem. Rev. 2004, 104, 2723.

13. Brindle, I. D.; Doyle, P. P. Can. J. Chem. 1983, 61, 1869.

14. (a) Benesch R. E; Benrsch R. J. Am. Chem. Soc 1958, 80, 1666 (b) Ran, R.; Pittman, U. C. J. Heterocyclic Chem. 1993, 30, 1673 and references cited therein.

15. Sharghi H.; Salimi Beni A. Synthesis 2004, 17, 2900.

16. Shamsipour, M.; Karami, A.; Yamini, Y.; Sharghi, H.; Salimi Beni A., J. Chem. Eng. Data 2003, 48, 1088.

17. Shamsipour, M.; Ershad, S.; Yari, A.; Sharghi, H.; Salimi Beni A., Analytical Sciences 2004, $20,1$.

18. Buchanan G. W.; Driega A. B.; Moghimi A.; Bensimon C.; Bourque K., Can. J. Chem. 1993, 71,951 . 